\title{
Texture of Material for Imitation Crab Meat Detected from Dynamic Behaviors
}

\author{
Teruo Nakayama,* Satoshi Kanoh,* and Eiji Niwa* \\ (Received January 6, 1988)
}

\begin{abstract}
Dynamic modulus and dynamic loss of the materials for imitation crab meat in foreign and domestic markets were predicted from the stress relaxation data with four-element model. The dependence of their dynamic modulus and dynamic loss on angular frequency was displayed by the computer. The experimental value of dynamic modulus at 18.84 radian/s was about $10 \%$ higher than the predicted value. However the experimental value of dynamic loss at 18.84 radian/ $s$ was almost 80 times as large as the predicted value. This fact suggests that another relaxation time would be included in the range of a very short time of our stress relaxation measurements, and that the stress corresponding to the $10 \%$ increment of experimentally obtained dynamic modulus, would be already relaxed during the application process of the constant strain in the stress relaxation measurement. From the comparison of dynamic and sensory data, we conclude that the experimental values of dynamic modulus and dynamic loss corresponded to the sensory softness. Especially the experimental value of dynamic loss was an important data, since it could not be predicted from stress relaxation data.
\end{abstract}

The imitation crab meat of shreded type ${ }^{1)}$ is familiar for dishes in Japan and also in foreign countries. This product is an imitation of each genuine single fiber separated from a bundle of muscle fibers of genuine carb leg. This is manufactured by cutting a large block of kamaboko (its size was $50 \mathrm{~cm} \times 70 \mathrm{~cm} \times 5 \mathrm{~cm}$ : we call it the material) into fiber-like strings. In the previous study, ${ }^{1)}$ we evaluated the material, instead of imitation crab meat itself, from stress relaxation and creep measurements. We predicted creep behavior from stress relaxation data. For the most part, this predicated creep curve fitted to the experimental points. Agreement was observed between the sensory evaluation and the stress relaxation (or creep) measurement.

Kishimoto et al., ${ }^{2,3)}$ Hirata, ${ }^{4)}$ and Takagi ${ }^{\text {b) }}$ investigated the dynamic behavior of kamaboko from damped oscillation experiments. They described that dynamic modulus, ${ }^{2)}$ dynamic viscosity, ${ }^{2)}$ and the ratio of dynamic loss to dynamic modulus ${ }^{4,5)}$ related to the quality of kamaboko. For the evaluation of the texture of imitation crab meat, the result of dynamic measurement might be additional important data to our static data. For the discussion of the relationship between stress relaxation data and dynamic data, it might be valuable to predict dynamic behavior from stress relaxation data. In this study, we measured dynamic modulus and dynamic loss, and also predicted them form streass relaxation data. Then we tried to compare the experimental value with the predicted value in the abovementioned viewpoint.

\section{Materials and Methods}

\section{Materials}

Four kinds of minced fish products (surimi based products) were purchased from marine food companies and were used for stress relaxation and dynamic measurements. Ingredient compositions of these products were already shown in Table 1 of previous paper. ${ }^{1 !}$

\section{Stress Relaration Measurement}

Stress relaxation behavior was measured by a rheometer (Rheoner RE 3305, Yamaden Co., Ltd.) at $19 \pm 1^{\circ} \mathrm{C}$. The procedures were already reported in previous paper. ${ }^{1)}$ The results of stress relaxation measurement reported in previous paper, ${ }^{1)}$ were used to predict dynamic behavior.

\section{Dynamic Measurement}

Dynamic modulus and dynamic loss were measured by Rheolograph Gel (Toyo Seiki Manufacturing Co., Ltd.) at $19 \pm 1^{\circ} \mathrm{C}$. A sample having the shape of square pillar $(1.3 \mathrm{~cm} \times 1.3 \mathrm{~cm}$

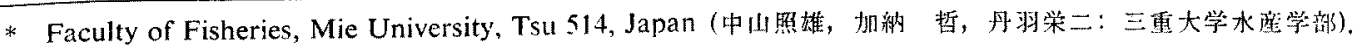


$\times 2.0 \mathrm{~cm}$ ) was cut off from each minced fish product by means of cutter blades set in parallel. The stress of $8700 \mathrm{dyn} / \mathrm{cm}^{2}$ or less was applied to hold the sample between top and bottom plates of Rheolograph Gel. Dynamic strain (strain amplitude $\mathbf{0 . 0 0 5}$, angular frequency 18.84 radian/s) was applied to the sample from the same direction as the extruding direction during the manufacturing process. This direction corresponded to the direction of the sample height of $2.0 \mathrm{~cm}$.

\section{Computer Graphics}

The derivation of predicted curve and the graphic display were done by the use of a personal computer (PC 9801 VX 21, Nihon Electric Co., Ltd.).

Sensory Evaluation and Plunger Penetration Test

The procedures were already reported in previous paper. ${ }^{12}$ The results reported in prevous paper ${ }^{1)}$ were used to discuss their relation to dynamic behavior.

\section{Results and Discussion}

The change of relaxation modulus $E(t)$ with time $t$ was expressed as:

$$
E(t)=E_{M 1} e^{-t / \tau_{M}}+E_{M / 2} e^{-t / \tau_{M H_{2}}}
$$

where $E_{M i}$ and $\tau_{M i}$ denote the elasticity of the spring, and the relaxation time, of the $i$ th Maxwell element, respectively $(i=1,2) .^{* 1}$ The relaxation time is defined by the following relation:

$$
\tau_{M i}=\frac{\eta_{M i}}{E_{M i}}
$$

where $\eta_{M i}$ denotes the viscosity of the dash-pot of the $i$ th Maxwell element.

The change of dynamic modulus $E^{\prime}(\omega)$ and dynamic loss $E^{\prime \prime}(\omega)$ with angular frequency $\omega$ was expressed as:

$$
\begin{gathered}
E^{\prime}(\omega)=E_{M 1} \frac{\omega^{2} \tau_{M 1}{ }^{2}}{1+\omega^{2} \tau_{M 1}{ }^{2}}+E_{M 2} \frac{\omega^{2} \tau_{M 2}{ }^{2}}{1+\omega^{2} \tau_{M 2}{ }^{2}} \\
E^{\prime \prime}(\omega)=E_{M 1} \frac{\omega \tau_{M 1}}{1+\omega^{2} \tau_{M 1}{ }^{2}}+E_{M 2} \frac{\omega \tau_{M 2}}{1+\omega^{2} \tau_{M 2}{ }^{2}}
\end{gathered}
$$

where $E_{M i}$ and $\tau_{M i}$ were the same symbols as defined in the above-mentioned relaxation modulus. ${ }^{* 2}$ Therefore to substitute Maxwellian rheological parameters calculated from relaxation modulus time responses for $E_{M 1}, \tau_{M 1}, E_{M 2}, \tau_{M 2}$ of eq (3) and eq (4), dynamic modulus $E^{\prime}(\omega)$ and dynamic loss $E^{\prime \prime}(\omega)$ were predicted.

According to the theory, when $\omega$ is equal to $1 / \tau_{M i}, E^{\prime}(\omega)$ varies in the steepest way in eq (3) and $E^{\prime \prime}(\omega)$ becomes the maximum in eq (4). The maximum value of $E^{\prime \prime}(\omega)$ is equal to one half of changeable quantity of $E^{\prime}(\omega)$ in the range of angular frequency $\omega$ around $1 / \tau_{M i}$. Therefore this phenomenon is called the mechanical dispersion.

The dependence of dynamic modulus and dynamic loss of minced fish products on angular frequency was displayed by the computer, as shown in Fig. 1 and 2. We magnified the angular frequency range below $0.005 \mathrm{radian} / \mathrm{s}$ in Fig. 1, and we magnified the angular frequency range between $0.005 \mathrm{radian} / \mathrm{s}$ and $1 \mathrm{radian} / \mathrm{s}$ in Fig. 2. As was expected, two maxima of the dynamic loss appeared. The angular frequencies for these maxima were shown in Table 1, together with the relaxation times already reported in the previous paper. ${ }^{1)}$ In accordance with the theory, each angular frequency for the maximum of dynamic loss was equal to the reciprocal of the corresponding relaxation time though two relaxation times were used in the analysis of our experiment. Therefore the product of this angular frequency

*1 M1 corresponds to Maxwell element of long relaxation time and M2 corresponds to that of short relaxation time.

*2 If the relaxation spectrum can be measured in wide range of time scales, we will calculate $E^{\prime}(\omega)$ and $E^{\prime \prime}(\omega)$ by the use of relaxation spectrum $E(\tau)^{0}$ as follows:

$$
\begin{aligned}
& E^{\prime}(\omega)=\int_{0}^{\infty} E(\tau) \frac{\omega^{2} \tau_{M^{2}}{ }^{2}}{1+\omega^{2} \tau_{M}^{2}} d \tau_{M} \\
& E^{\prime \prime}(\omega)=\int_{0}^{\infty} E(\tau) \frac{\omega \tau_{M}}{1+\omega^{2} \tau_{M}{ }^{2}} d \tau_{M}
\end{aligned}
$$

But in the case of minced fish products it is impossible to measure the relaxation spectrum covering wide range of time scales, because of the unstability of minced fish products during the measurements and the accuracy limit of measuring apparatus. Therefore we calculated two relaxation times in the limited time scale range of stress relaxation measurement. In this report, $E^{\prime}(\omega)$ and $E^{\prime \prime}(\omega)$ were calculated by the use of these two relaxation times (Refer to eq (3) and eq (4)). 


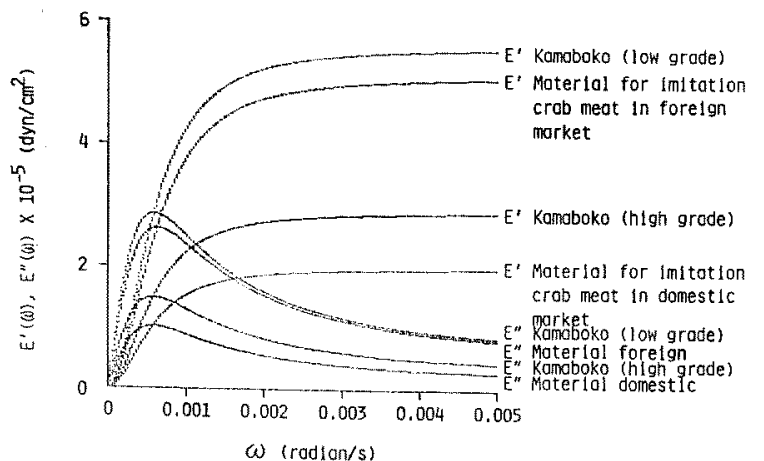

Fig. 1. Computer prediction of dynamic behavior of minced fish products in the angular fre. quency range below 0.005 radian/s.

$\omega$ : angular frequency

$E^{\prime}(\omega)$ : dynamic modulus

$E^{\prime \prime}(\omega)$ : dynamic loss

The curves are drawn by substituting the parameters of the four-element model of relaxation modulus $E(t)$ for eq (3) and eq (4).

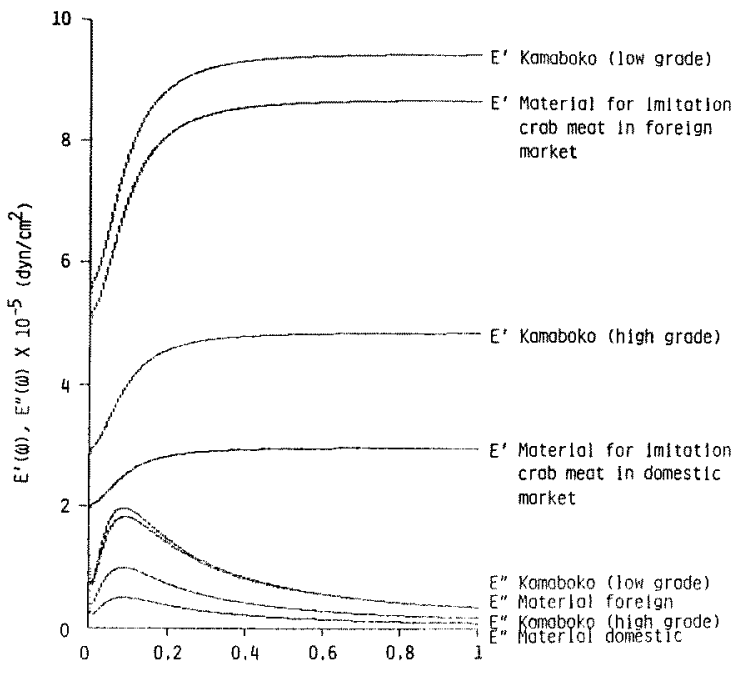

Fig. 2. Computer prediction of dynamic behavior of minced fish products in the angular frequency range below $1 \mathrm{radian} / \mathrm{s}$.

$\omega$ : angular frequency

$E^{\prime}(\omega)$ : dynamic modulus

$E^{\prime \prime}(\omega)$ : dynamic loss

The curves are drawn by substituting the parameters of the four-element model of relaxation modulus $E(t)$ for eq (3) and eq (4).

by the corresponding relaxation time was exactly one.

When $(1)$ is equal to $1 / \tau_{M 1}$ in eq (4) (Refer to $\omega_{\perp}$ in Table 1), the first term of eq (4) becomes (1/2) $E_{A 1}$ and the second term becomes negligible. When $\omega$ is equal to $1 / \tau_{M 2}$ in eq (4) (Refer to $\omega_{2}$ in Table $1)$, the second term of eq (4) becomes $(1 / 2) E_{M 2}$ and the first term becomes negligible.
In accordance with the above theory, one maximum of $E^{\prime \prime}(\omega)$ was equal to the value of $(1 / 2) E_{M 1}$ (Fig. 1 and Table 2), and another maximum of $E^{\prime \prime}(\omega)$ was equal to the value of $(1 / 2) E_{M 2}$ (Fig. 2 and Table 2).

In the dynamic measurements, the frequency of $3 \mathrm{cycle} / \mathrm{s}$ was applied to the sample. This frequency corresponded to the angular frequency of 
Table 1. Angular frequencies for two maxima of dynamic loss and their corresponding relaxation times

\begin{tabular}{|c|c|c|c|c|}
\hline Minced fish products & $\begin{array}{c}\text { Angular frequency } \\
\omega_{1} \text { where } E^{\prime \prime}(\omega) \\
\text { becomes the maximum } \\
\text { (radian/s) }\end{array}$ & $\stackrel{\tau_{M 1}}{(s)}$ & $\begin{array}{c}\text { Angular frequency } \\
\omega_{2} \text { where } E^{\prime \prime}(\omega) \\
\text { becomes the maximum } \\
\text { (radian/s) }\end{array}$ & $\tau_{M 2}$ \\
\hline $\begin{array}{l}\text { Material for imitation carb meat in } \\
\text { domestic market }\end{array}$ & $0.00059^{* 1}$ & $1700^{* 2}$ & $0.100^{* 3}$ & $10^{* 2}$ \\
\hline Kamaboko (high grade) & $0.00059 * 1$ & $1700 * 2$ & $0.091^{* 3}$ & $11 * 2$ \\
\hline $\begin{array}{l}\text { Material for imitation carb meat in } \\
\text { foreign market }\end{array}$ & $0.00063^{* 1}$ & $1600 * 2$ & $0.100^{* 3}$ & $10^{* 2}$ \\
\hline Kamaboko (low grade) & $0.00059 * 1$ & $1700 * 2$ & $0.091^{* 3}$ & $11^{* 2}$ \\
\hline
\end{tabular}

*1 This value was found in Fig. 1 .

*2 This value was obtained in stress relaxation measurement of our previous paper.

* 3 This value was found in Fig. 2.

Table 2. Two maxima of dynamic loss and their corresponding Maxwellian elasticities

\begin{tabular}{|c|c|c|c|c|}
\hline Minced fish products & $\begin{array}{l}E^{\prime \prime}\left(\omega_{1}\right) \times 10^{-5} \\
\quad\left(\text { dyn } / \mathrm{cm}^{2}\right)\end{array}$ & $\begin{array}{l}\frac{1}{2} E_{H 1} \times 10^{-5} \\
\left(\mathrm{dyn} / \mathrm{cm}^{2}\right)\end{array}$ & $\begin{array}{c}E^{\prime \prime}\left(\omega_{2}\right) \times 10^{-5} \\
\left(\mathrm{dyn} / \mathrm{cm}^{2}\right)\end{array}$ & $\begin{array}{c}\frac{1}{2} E_{M 2} \times 10^{-5} \\
\left(\mathrm{dyn} / \mathrm{cm}^{2}\right)\end{array}$ \\
\hline $\begin{array}{l}\text { Material for imitation } \\
\text { crab meat in domestic } \\
\text { market }\end{array}$ & $1.0^{* 1}$ & $1.0 * 2$ & $0.5^{* 3}$ & $0.5^{* 2}$ \\
\hline Kamaboko (higl grade) & $1.5^{* \downarrow}$ & $1.5^{* *}$ & $1.0^{* 3}$ & $1.0^{* 2}$ \\
\hline $\begin{array}{l}\text { Material for imitation } \\
\text { crab meat in foreign } \\
\text { market }\end{array}$ & $2.6^{* 1}$ & $2.6 * 2$ & $1.8^{* 3}$ & $1.8 * 2$ \\
\hline Kamaboko (low grade) & $2.9 * 1$ & $2.9 * 2$ & $2.0^{* 3}$ & $2.0^{* 2}$ \\
\hline
\end{tabular}

*1 This value was found in Fig. 1 .

*2 This value was obtained in stress relaxation measurement of out previous paper

*3 This value was found in Fig. 2.
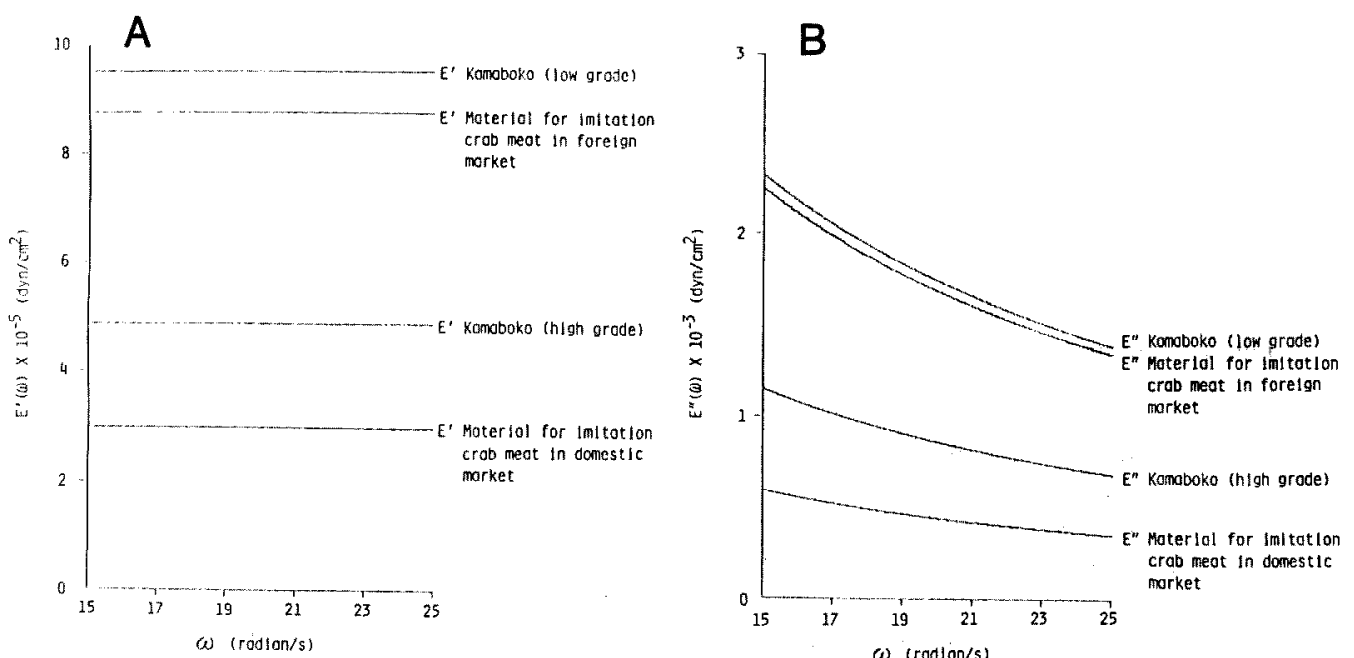

Fig. 3. Computer prediction of dynamic modulus (A) and dynamic loss (B) of minced fish products in the angular frequency range between 15 radian $/ \mathrm{s}$ and $25 \mathrm{radian} / \mathrm{s}$.

$\omega:$ angular frequency

$E^{\prime}(\omega)$ : dynamic modulus

$E^{\prime \prime}(\omega)$ : dynamic loss

The curves are drawn by substituting the parameters of the four-element model of relaxation modulus $E(t)$ for req (3) and eq (4). 
Table 3. Predicted and experimentally obtained values of dynamic modulus and dynamic loss

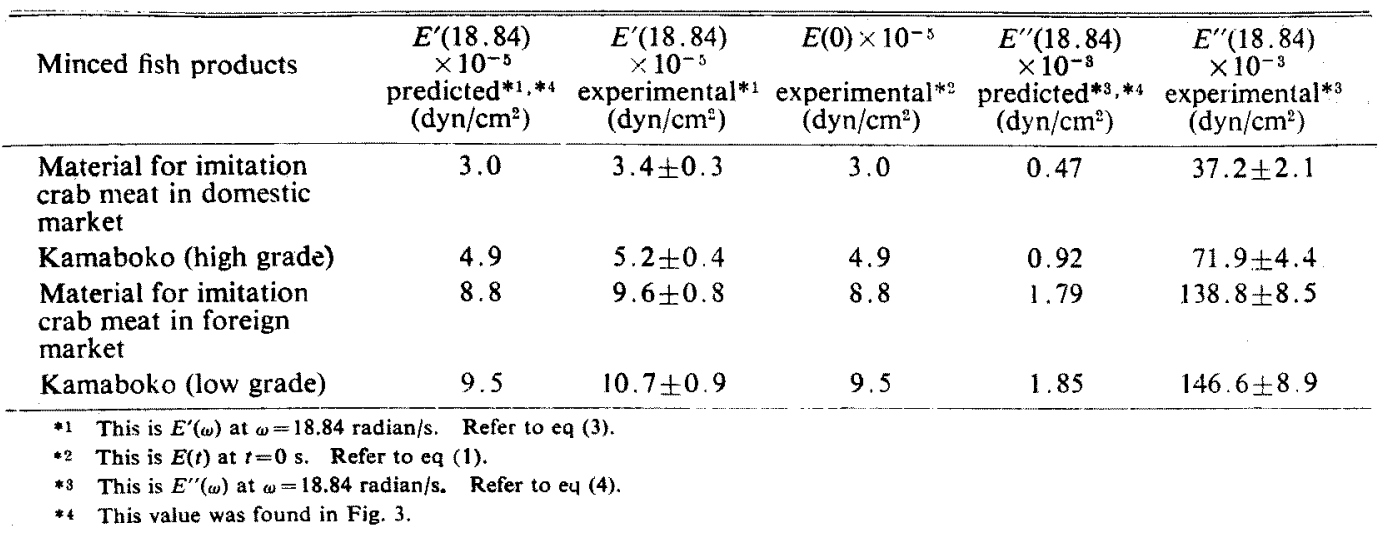

$18.84 \mathrm{radian} / \mathrm{s}$. We compared the experimental value of dynamic modulus $E^{\prime}(18.84)$ with the predicted value. The experimental value was about $10 \%$ higher than the predicted value, consistently (Table 3). Around the angular frequency of $18.84 \mathrm{radian} / \mathrm{s}$, the predicted curve of dynamic modulus $E^{\prime}(\omega)$ almost did not depend on angular frequency $\omega$ (A of Fig. 3). We also compared the experimental value of dynamic loss $E^{\prime \prime}(18.84)$ with the predicted value. In this case, the experimental value was about 80 times the predicted value (Table 3 ).

We considered, at first, the reason why the experimental value of dynamic modulus was about $10 \%$ higher than the predicted value.

In the stress relaxation measurements, an infinitely high speed is advisable theoretically for the application of the constant strain. But when the speed of $10 \mathrm{~mm} / \mathrm{s}$ was used for the application of the constant stress in the creep measurements, ${ }^{1}$ ) the undesirable shock vibration was observed. Therefore to avoid the shock we used the speed of $5 \mathrm{~mm} / \mathrm{s}$, as well, for the application of the constant strain in the stress relaxation measurements. In the dynamic measurements the deformation amplitude was $\pm 0.1 \mathrm{~mm}$ and the frequency was 3 cycle/s. Therefore the deformation speed of the sample was $0.6 \mathrm{~mm} / \mathrm{s}$. The speed $5 \mathrm{~mm} / \mathrm{s}$ used for the application of the constant strain in the stress relaxation measurements, was adequately larger than the deformation speed $0.6 \mathrm{~mm} / \mathrm{s}$ in the dynamic measurements. Therefore the time scale $(3 \mathrm{cycle} / \mathrm{s})$ of dynamic measurements was included in the short time range of the stress relaxation measurements.
In the stress relaxation measurements the applied constant strain ranged between 0.02 and 0.12 . This strain was located within the linear viscoelastic region. ${ }^{1)}$ In the dynamic measurements the strain amplitude was 0.005 . The deformation was much smaller in the dynamic measurements. Therefore this strain amplitude was located of course within the linear viscoleastic region.

These facts do not seem to be the reason of the difference of dynamic modulus between the experimental value and the predicted value.

In the stress relaxation measurements, the applied stress for holding the sample was completely relaxed just before the measurement. In the dynamic measurements the stress of $8700 \mathrm{dyn} /$ $\mathrm{cm}^{2}$ or less was applied to hold the sample between top and bottom plates of Rheolograph Gel, and the dynamic measurements started during the stress relaxation process. However, the measurable dependence of the value of dynamic modulus $E^{\prime}(18.84)$ on the magnitude of this applied stress was not detected. Therefore the applied stress would not be the main reason of $10 \%$ higher value of experimental dynamic modulus.

In the next place, we tried to consider the reason why the experimental value of dynamic loss was much larger than the predicted value. The experimental value of dynamic loss $\mathrm{E}^{\prime \prime}(18.84)$ ranged between $0.4 \times 10^{5}$ and $1.5 \times 10^{5} \mathrm{dyn} / \mathrm{cm}^{2}$ (Table 3). The magnitude of these values was similar to the magnitude of two maxima of dynamic loss displayed by the computer (Fig. 1, Fig. 2, Table 2). Therefore it is reasonable to suggest that the third maximum of dynamic loss exists

* $(0.1 \mathrm{~mm} \times 2) \times 3 \mathrm{cycle} / \mathrm{s}=0.6 \mathrm{~mm} / \mathrm{s}$. Sample was elongated $0.1 \mathrm{~mm}$ and was compressed $0.1 \mathrm{~mm}$. Therefore $0.1 \mathrm{~mm}$ was multiplied by 2 . 
around angular frequency of $18.84 \mathrm{radian} / \mathrm{s}$. Supposing dynamic loss is maximum at this angular frequency, its corresponding relaxation time becomes $0.053 \mathrm{~s}$. This relaxation time corresponds to very short time of our stress relaxation measurements.

In the next place, we considered whether the third relaxation mechanism was included in the instantaneous modulus $E(0)$ of stress relaxation measurements. If the difference of dynamic modulus $E^{\prime}(18.84)$ between the experimental value and the predicted value was equal to twice experimental dynamic loss $E^{\prime \prime}(18.84)$, we could conclude that the third relaxation mechanism was not caught in the stress relaxation measurements. Contrary to expectation, this difference was much smaller than twice experimental dynamic loss $E^{\prime \prime}(18.84)$. Therefore we think that the third relaxation mechanism was caught also in the stress relaxation measurements. However it appeared in the range of such a short time that we could not recognize it in the analysis of stress relaxation data. The reason is due to the fact that the accuracy of stress relaxation curve is rather low in the range of very short time.

In this step, we reconsidered the reason why the experimental value of dynamic modulus was about $10 \%$ higher than the predicted value. In the stress relaxation measurements, the constant strain $(0.02,0.04,0.06,0.08,0.10$, or 0.12$)$ was applied by the speed of $5 \mathrm{~mm} / \mathrm{s}$. It takes $0.067 \mathrm{~s}$ to attain the strain of 0.02 , and $0.402 \mathrm{~s}$ to attain the strain of 0.12 . These time scales required to attain the constant strain, were nearly equal to or larger than the above-mentioned relaxation time $(0.053 \mathrm{~s})$ corresponding to the dynamic measurement. Therefore we deduce that the stress corresponding to the $10 \%$ increment of experimentally obtained dynamic modulus, would be already relaxed during the application process of the constant strain in the stress relaxation mesaurement.

In this series of papers, we used the mechanical model of four elements. In the analysis of stress relaxation data, we used best-fit procedure." This method is effective to detect the difference in the shape of stress relaxation curves. However this method is still qualitative because there is a relaxation spectrum in truth, and the stress relaxation curve is not accurate in the range of very short time because of the accuracy limit of the measuring apparatus. In the case of dynamic measurements, we can easily obtain the informa- tion of short time range. In this paper, we found the discrepancy between the results of stress relaxation and dynamic measurements in the use of four-element model. As already discussed in the footnote of previous section, it is impossible to measure the relaxation spectrum covering wide range between very short time and very long time. Therefore in this meaning both stress relaxation data and dynamic data are still important to discuss the texture of minced fish products.

In this place, we consider the relationship between the result of dynamic measurement and the result of sensory evaluation.

The experimental values of $E^{\prime}(18.84)$ and $E^{\prime \prime}(18.84)$ were small in material for imitation crab meat in domestic market. They were medium in kamaboko (high grade), and large in both material for imitation crab meat in foreign market and kamaboko (low grade) (Table 3).

Material for imitation crab meat in domestic market acquired the highest scores in both the sense intensity of jelly strength and the sensory softness (Table 5 of previous paper ${ }^{1)}$ ). Kamaboko (high grade) acquired high scores in these categories. Both material for imitation crab meat in foreign market and kamaboko (low grade) acquired low scores in those categories.

In plunger penetration test presented in the previous paper, ${ }^{1)}$ materials for imitation crab meat in both domestic and foreign markets and kamaboko (low grade) had the jelly strength of similar values. Kamaboko (high grade) only had the jelly strength of large values. In the breaking deformation, kamaboko (high grade) also had large values, material for imitation crab meat in domestic market had medium values, and both material for imitation crab meat in foreign market and kamaboko (low grade) had small values.

From the above-mentioned resuits, we conclude that the experimental values of $E^{\prime}(18.84)$ and $E^{\prime \prime}(18.84)$ corresponded to the sensory softness even if the instrumental value of jelly strength did not correspond to the sense intensity of jelly strength.

Especially the experimental value of $E^{\prime \prime}(18.84)$ was additional important data to the static data, since it could not be predicted correctly from $E(t)$. And the relative difference between minced fish products was rather clearer in experimental $E^{\prime \prime}(18.84)$ than in experimental $E^{\prime}(18.84)$ (Table $3)$. 


\section{References}

1) T. Nakayama, S. Kanoh, and E. Niwa: Nippon Suisan Gakkaishi, 54, 717-724 (1988).

2) A. Kishimoto and E. Maekawa: Nippon Suisan Gakkaishi, 28, 803-808 (1962).

3) A. Kishimoto and S. Hirata: Nippon Suisan
Gakkaishi, 29, 146-150 (1963).

4) S. Hirata: Nippon Suisan Gakkaishi, 30, 635638 (1964).

5) I. Takagi: Nippon Suisan Gakkaishi, 39, 661665 (1973).

6) T. Nakagawa: Rheology 2nd ed., Iwanami Shoten, Tokyo, 1978, pp. 1-183.

Nippon Suisan Gakkaishi : Formerly Bull. Japan. Soc. Sci. Fish. 